\title{
Nitrogen isotope fractionation between metallic melt and silicate melt
}

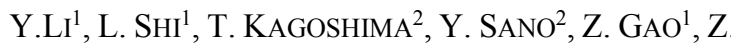

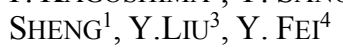

${ }^{1}$ Guangzhou Institute of Geochemistry, Chinese Academy of Sciences, Guangzhou 510640, China (Yuan.Li@gig.ac.cn)

${ }^{2}$ Division of Ocean-Earth System Science, Atmosphere and Ocean Research Institute, University of Tokyo, Kashiwa, Chiba 277-8564, Japan

${ }^{3}$ Institute of Geochemistry, Chinese Academy of Sciences, Guiyang 550081, China

${ }^{4}$ Geophysical Laboratory, Carnegie Institution of Washington, Washington DC 20015, USA

The origin of planetary volatiles such as nitrogen and carbon is critical for understanding planetary accretion, differentiation, and habitability. The large nitrogen isotope fractionation among cosmochemical reservoirs can provide critical constraints on volatile accretion. We performed highpressure experiments to investigate the effects of temperature, oxygen fugacity, and compositions of metallic melt and silicate melt on the element partitioning and isotope fractionation of nitrogen between Fe-rich metallic melt and silicate melt at 1-8 GPa, using a piston cylinder and multianvil apparatus. The experimental oxygen fugacity was varied between $\sim \mathrm{IW}-4.7$ and IW-0.3, covering the redox conditions of planetary magma oceans in the inner solar system. We will show that the metal/silicate nitrogen partition coefficent is controlled by oxygen fugacity, temperature, pressure, and compositions of silicate melt and metallic melt, consistent with previous results. We will further show that the metal/silicate nitrogen isotope fractionation factor increases from $-4 \%$ to $+10 \%$ with oxygen fugacity decreasing from 0.3 to $4.7 \mathrm{log}$ units below the iron-wüstite buffer. Applying these $\mathrm{N}$ isotope fractionation data to planetary core-mantle segregation, we find that that the nitrogen isotopes do not fractionate significantly between Earth's core and mantle even if a large fraction of the Earth's nitrogen were sequestered into Earth's core. The Earth's mantle nitrogen isotope composition and the superchondritic carbon/nitrogen ratio may be explained by an early accretion of enstatite chondrite-like materials, followed by a post-giant impact accretion of differentiated planetesimals with carbonaceous chondrite-like compositions. 